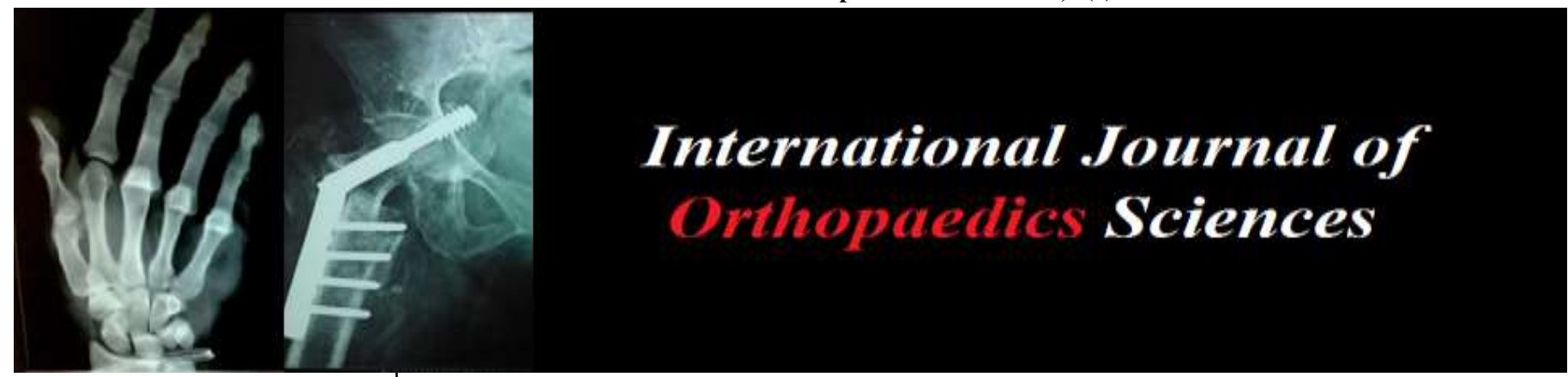

E-ISSN: 2395-1958

P-ISSN: 2706-6630

IJOS 2021; 7(2): 689-697

(C) 2021 IJOS

www.orthopaper.com

Received: 17-02-2021

Accepted: 23-03-2021

Dr. Prakashappa TH MBBS, D'ortho, MS (Ortho)

Sanjay Gandhi Institute of

Trauma and Orthopaedics,

Byrasandra, Bengaluru,

Karnataka, India

Dr. Sindhu B

MBBS, Resident in MS (Ortho),

Sanjay Gandhi Institute of

Trauma and Orthopaedics,

Byrasandra, Bengaluru,

Karnataka, India

Dr. Bharat Raja BS

MBBS, D'ortho, MS (Ortho),

Sanjay Gandhi Institute of

Trauma and Orthopaedics,

Byrasandra, Bengaluru,

Karnataka, India

Dr. Easwar B

MBBS, Resident in MS (Ortho), Resident in Orthopedic, Sanjay Gandhi Institute of Trauma and Orthopaedics, Byrasandra, Bengaluru, Karnataka, India
Corresponding Author: Dr. Sindhu B

MBBS, Resident in MS (Ortho),

Sanjay Gandhi Institute of

Trauma and Orthopaedics,

Byrasandra, Bengaluru,

Karnataka, India

\section{Comparative study of short proximal femoral nail versus long proximal femoral nail in the management of stable intertrochanteric fractures}

\author{
Dr. Prakashappa TH, Dr. Sindhu B, Dr. Bharat Raja BS and Dr. Easwar \\ B
}

DOI: https://doi.org/10.22271/ortho.2021.v7.i2i.2693

\section{Abstract}

Aim: This study is intended to compare the clinical outcome of short proximal femoral nail vs long proximal femoral nail in the management of stable intertrochanteric fractures.

Methods: This is a prospective comparative study of 40 cases of fresh stable intertrochanteric femur fractures admitted to Sanjay Gandhi Institute of Trauma and Orthopedics Bangalore between December 2018 to September 2019. The study population were randomly divided into 2 groups with 20 patients in each group. Group A: The patients who were operated with Short Proximal Femoral Nail. Group B: The patients who were operated with Long Proximal Femoral Nail. Cases were considered according to inclusion and exclusion criteria. Results were evaluated by fracture union on X-ray and Modified Harris hip score.

Results: In our series, majority of the cases in short PFN group were males, i.e. 15 (75\%) and equal population of males and females in long PFN group, maximum age of 80 years and minimum age of 18 years, most of the patients were between 40 to 60 years. Mean age of 55.6 years in short PFN group and 56.6 in long PFN group, $85 \%$ of cases were admitted due to slip and fall. Functional outcome was evaluated by Modified Harris hip score at 3 months, 6 months and 1 year follow up. In short PFN group 9 (45\%) patients had excellent outcome, 8 (40\%) patients had good outcome, 2 (10\%) patients had fair outcome, $1(5 \%)$ patient had poor outcome. In long PFN group 8 (40\%) patients had excellent outcome, 9 (45\%) patients had good outcome, 3 (15\%) patients had fair outcome.

Conclusion: Use of Long PFN has advantages over short PFN in terms of the less postoperative complications like peri implant fracture and anterior thigh pain \& better functional outcome. The terms of successful outcome include a good understanding of fracture biomechanics, proper patient selection, good preoperative planning and accurate instrumentation.

Keywords: PFN, intertrochanteric, stable, short PFN, long PFN

\section{Introduction}

Proximal femoral fractures are most devastating injury. They commonly affect the elderly. The frequency of these proximal femoral fractures has increased primarily due to the increasing life span and more sedentary life style. Intertrochanteric fractures constitute about $50 \%$ of all proximal femoral fractures. $90 \%$ of intertrochanteric fractures occur in elderly as a result of trivial trauma due to associated osteoporosis. IT fractures in younger individuals is a result of high velocity trauma ${ }^{[1]}$.

Operative treatment is the best option in trochanteric fractures ${ }^{[2]}$. Conventional implants like dynamic hip screw, angular blade plates or cephalo medullary nails can be used for the successful treatment of these fractures ${ }^{[3]}$. The theoretical advantages proposed of the nail include percutaneous insertion and improved fracture fixation biomechanics $\left[\begin{array}{ll}4 & 5\end{array}\right.$. Biomechanically very large force is required to produce the medial displacement of femoral shaft with intramedullary device which is a common complication of extramedullary devices ${ }^{[6]}$. The use of intramedullary devices allows a faster restoration of postoperative walking ability, when compared with extramedullary sliding devices ${ }^{[7]}$.

Most commonly used intramedullary devices for the management of IT femur fractures are Gamma nail and Short PFN. 
There are different studies available in literature claiming superiority of Gamma nail [8, 9] and Short PFN [10-12] individually. Among Short PFN and Gamma nail, Short PFN had shown either equal results ${ }^{[13]}$ or better results [14] biomechanically in the management of unstable intertrochanteric fractures. Both implants have higher rate of complications like anterior thigh pain, femoral shaft fracture distal to the tip of nail ${ }^{[15-18]}$ which may lead to higher rate of revision surgery ${ }^{[19]}$. Few studies have shown that long intramedullary devices may overcome these complications ${ }^{[20]}$. In this study long and short PFN are used for stable IT femur fractures. Need for this study is to compare the results of Long PFN against Short PFN with regard to pain, walking ability using Harris Hip Score, post-operative complications, mean time for radiological union and failure rates.

\section{Aims and objectives of the study}

- This study is intended to compare the clinical outcome of short proximal femoral nail vs long proximal femoral nail in the management of stable intertrochanteric fractures.

- To evaluate the advantages, disadvantages and possible complications associated with fixation of IT fractures with long and short PFN.

\section{Methodology}

Patients brought to the Sanjay Gandhi institute of trauma and orthopedics with stable intertrochanteric femoral fractures from December 2018 to September 2019 fulfilling the inclusion and exclusion criteria were taken into the study after obtaining written informed consent. 40 cases were considered.

\section{Inclusion criteria}

- Adult patients of both sexes aged between 18-80 years.

- All traumatic stable intertrochanteric fractures.

- Patients willing for treatment and giving informed written consent for necessary investigations and surgery.

\section{Exclusion criteria}

- Open fractures.

- Associated ipsilateral fractures like distal femur and proximal tibial fractures.

- Pathological fractures.

- Pre- existing diseases or deformities of injured hip.

- Cases with late presentation to the hospital, old neglected fractures.

- Patients unfit for surgery.

- Unstable intertrochanteric fractures.

After the patient with intertrochanteric fracture was admitted to hospital all the necessary clinical details were recorded and proforma was prepared for this study. Demographic data, detailed history, clinical examination, details of investigations and interventions was recorded in the study proforma. Patients were randomly divided into 2 groups
Group 1: Patients who were planned to be treated with short PFN.

Group 2: Patients who were planned to be treated with long PFN.

Emergency management of all life threatening conditions was carried out in casualty with respect to airway breathing circulation. Affected limb was immobilised in BB splint by skin traction and skeletal traction was used if surgery is delayed. After stabilisation of vitals radiographs of the affected extremities were carried out. Routine X-Ray pelvis with bilateral hip AP and $\mathrm{x}$ ray of affected thigh full length antero posterior view and lateral view was taken to know the details of the fracture. The fracture pattern was classified. The fracture was immobilized using skin traction with weight. Written informed consent of patient \& relatives for internal fixation taken. All the patients were kept nil per oral overnight. Clinical and radiological measurement of proper nail size was carried out. Patients were randomly divided into 2 groups and were treated with long and short PFN with 20 patients in each group.

\section{Post-operative protocol}

a. Patients pulse, blood pressure, respiration, temperature was monitored

b. Limb elevation over pillow and patient kept under observation in recovery room until stable then shifted to ward.

c. IV antibiotics were continued for first 3 days and then it was shifted to oral.

d. Blood transfusion was given depending on the requirement.

e. Static quadriceps exercises were started on 1st postoperative day.

f. Active quadriceps and hip flexion exercises were started on 3rd postoperative day.

g. Dressing was done at $2^{\text {nd }}, 6^{\text {th }}$ and $10^{\text {th }}$ postoperative day.

h. Sutures were removed on 12 th postoperative day.

i. Patients were advised to walk with partial weight bearing as soon as tolerable.

j. Full weight bearing walking was allowed after assessing for radiological and clinical union.

\section{Discharge}

Patients were discharged from the hospital when they were able to ambulate independently with or without walking aids.

\section{Follow up}

Radiological examination was repeated post operatively and patients were followed up at 6 weeks, 12 weeks, 6 months, 12 months. Healing was judged by both clinically- pain and motion at fracture site and radiological -bridging callus filling the fracture site or trabeculations across the fracture site and functional outcome was reviewed according to the Harris Hip score.

Table 1: Modified Harri's hip scoring system

\begin{tabular}{|c|c|}
\hline Pain & \\
\hline None or ignores it & 44 \\
\hline Slight, occasional, no compromise in activities & 40 \\
\hline - $\quad$ Mild pain, no effect on average activities, rarely moderate pain with unusual activities; may take aspirin. & 30 \\
\hline $\begin{array}{c}\text { - Moderate pain, tolerable but makes concession to pain. Some limitation of ordinary activity or work. May } \\
\text { require occasional pain medication stronger than aspirin. }\end{array}$ & 20 \\
\hline - $\quad$ Marked pain, serious limitation of activities. & 10 \\
\hline - $\quad$ Totally disabled, crippled, pain in bed, bed ridden & 0 \\
\hline Limp & \\
\hline
\end{tabular}




\begin{tabular}{|c|c|}
\hline None & 11 \\
\hline Slight & 8 \\
\hline - $\quad$ Moderate & 5 \\
\hline severe & 0 \\
\hline \multicolumn{2}{|l|}{ Support } \\
\hline None & 11 \\
\hline - $\quad$ Cane for long walks & 7 \\
\hline - $\quad$ Cane most of the time & 5 \\
\hline - $\quad$ One crutch & 3 \\
\hline - $\quad$ Two canes & 2 \\
\hline - $\quad$ Two crutches or not able to walk & 0 \\
\hline \multicolumn{2}{|l|}{ Distance walked } \\
\hline - Unlimited & 11 \\
\hline - $\quad$ Six blocks & 8 \\
\hline - $\quad$ Two or three blocks & 5 \\
\hline - $\quad$ Indoors only & 2 \\
\hline - $\quad$ Bed and chair only & 0 \\
\hline \multicolumn{2}{|l|}{ Sitting } \\
\hline - $\quad$ Comfortably in ordinary chair for 1 hour & 5 \\
\hline - $\quad$ On a high chair for 30 minutes & 3 \\
\hline - Unable to sit comfortably on any chair & 0 \\
\hline \multicolumn{2}{|l|}{ Enter public transportation } \\
\hline \multicolumn{2}{|l|}{ - $\quad$ Yes (1) No (0) } \\
\hline \multicolumn{2}{|l|}{ Stairs } \\
\hline - $\quad$ Normally without using railing & 4 \\
\hline - Normally using a railing & 2 \\
\hline - In any manner & 1 \\
\hline - Unable to do stairs & 0 \\
\hline \multicolumn{2}{|l|}{ Put on shoes and socks } \\
\hline - With ease & 4 \\
\hline - $\quad$ With difficulty & 2 \\
\hline - Unable & 0 \\
\hline Deformity (all yes $=4$, less than $4=0$ ) & \\
\hline - $\quad$ Less than 30 degree fixed flexion contracture & Yes \\
\hline - $\quad$ Less than 10 degree fixed abduction & Yes \\
\hline - $\quad$ Less than 10 degree fixed internal rotation in extension & Yes \\
\hline - $\quad$ Limb length discrepancy less than $3.2 \mathrm{cms}$ & Yes \\
\hline
\end{tabular}

\section{Range of motion (* indicates normal)}

Flexion $\left(* 140^{\circ}\right)$

Abduction $\left(* 40^{\circ}\right)$

Adduction $\left(* 40^{\circ}\right)$

External Rotation $\left(* 40^{\circ}\right)$

Internal Rotation $\left(* 40^{\circ}\right)$

\section{Range of Motion Scale}

$211^{\circ}-300^{\circ}$

$161^{\circ}-210^{\circ}$

$101^{\circ}-160^{\circ}$

$61^{\circ}-100$

$31^{\circ}-60^{\circ}$
(5)

(1) $0^{\circ}-30^{\circ}$

(0)

Table 2: Total Harris hip score

\begin{tabular}{|c|c|}
\hline Score & Rating \\
\hline $90-100$ & Excellent \\
\hline $80-89$ & Good \\
\hline $70-79$ & Fair \\
\hline$<70$ & poor \\
\hline
\end{tabular}

\section{Observation and results}

In our series, majority of the cases in short PFN group were males, i.e $15(75 \%)$ and equal population of males and females in long PFN group.

Table 2: Table showing gender distribution

\begin{tabular}{|c|c|c|c|c|c|c|}
\hline & & \multicolumn{2}{|c|}{ Implant used } & \multirow{2}{*}{ Total } & \multicolumn{2}{|c|}{ Chi square test } \\
\hline & & Short & Long & & Chi square value & p-value \\
\hline \multirow{4}{*}{ Sex } & 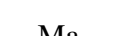 & 15 & 10 & 25 & \multirow{4}{*}{2.67} & \multirow{4}{*}{$0.10(\mathrm{NS})$} \\
\hline & Ma & $75.0 \%$ & $50.0 \%$ & $62.5 \%$ & & \\
\hline & \multirow{2}{*}{ Female } & 5 & 10 & 15 & & \\
\hline & & $25.0 \%$ & $50.0 \%$ & $37.5 \%$ & & \\
\hline
\end{tabular}

In our study mean age group of short PFN was 55 and mean age group of long PFN group was 56. 
Table 3: Table showing age distribution

\begin{tabular}{|c|c|c|c|c|c|c|c|c|c|c|}
\hline & \multirow{2}{*}{ Implant Used } & \multirow{2}{*}{$\mathbf{N}$} & \multirow{2}{*}{ Mean } & \multirow{2}{*}{ SD } & \multirow{2}{*}{$\begin{array}{c}\text { Mean } \\
\text { Difference }\end{array}$} & \multicolumn{2}{|c|}{ 95\% Confidence Interval of the Difference } & \multirow[b]{2}{*}{$\mathbf{t}$} & \multirow{2}{*}{ df } & \multirow{2}{*}{ p-value } \\
\hline & & & & & & Lower & Upper & & & \\
\hline \multirow{2}{*}{ Age } & Short & 20 & 55.60 & 10.71 & \multirow{2}{*}{-1.00} & \multirow{2}{*}{-9.00} & \multirow{2}{*}{7.00} & \multirow{2}{*}{-0.25} & \multirow{2}{*}{38} & \multirow{2}{*}{$0.80(\mathrm{NS})$} \\
\hline & Long & 20 & 56.60 & 14.05 & & & & & & \\
\hline
\end{tabular}

Independent sample t test $* p<0.05$ statistically Significant, $p>0.05$ Non Significant, NS

16 cases $(80 \%)$ affected were due to direct impact following trivial fall, 4 cases $(20 \%)$ due to road traffic accident in short PFN group, 18 cases ( $90 \%$ ) affected were due to direct impact following trivial fall, 2 cases (10\%) due to road traffic accident in long PFN group. According to the Boyd and Griffin classification, out of 20 cases in short PFN group 7 cases were of type 1, 13 were of type 2 and out of 20 cases in long PFN group 11 were of type 1 and 9 were of type 2.

\section{Operative time}

Long PFN took a longer operative time, mean of 55.15 minutes, compared to a mean of 50.50 minutes for performing a Short PFN surgery

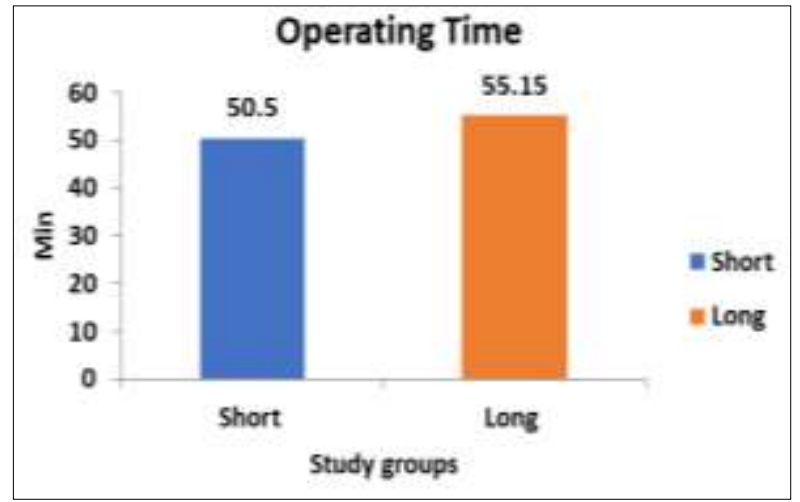

Chart 1: chart showing operative time
Intra operative blood loss

No statistically significant difference in blood loss between long and short PFN group

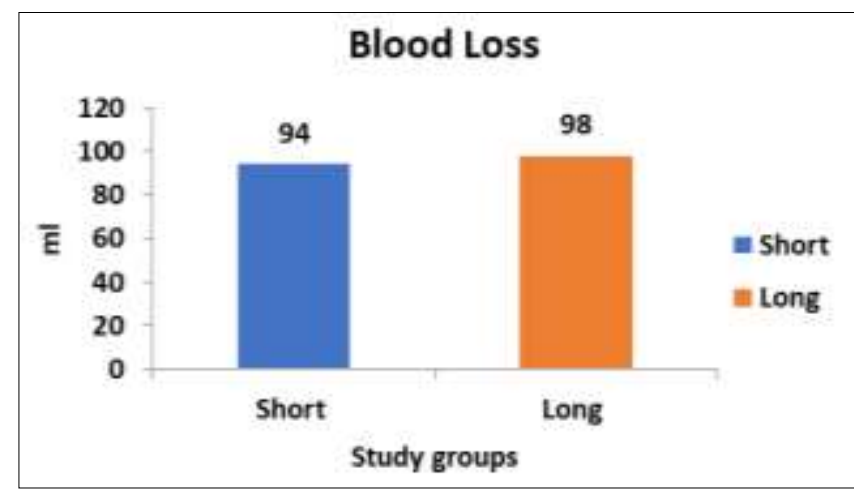

Chart 2: chart showing intra operative blood loss

Mean duration of hospital stay was 9.05 days for short PFN group and 8.75 days for long PFN group. In our study the mean time for union was 14.9 weeks in short PFN group and 16.05 weeks in long PFN group.

Modified Harris hip score at 1 year follow UP.

Mean Harris hip score in short PFN group at 1 year follow up was 85.75 and score for long PFN group at 1 year follow up was 90.75 which had a statistically significant difference.

Table 4: Table showing modified Harris hip score at 1 year follow up

\begin{tabular}{|c|c|c|c|c|c|c|c|c|c|c|}
\hline & \multirow[t]{2}{*}{ Implant Used } & \multirow[t]{2}{*}{$\mathbf{N}$} & \multirow[t]{2}{*}{ Mean } & \multirow[t]{2}{*}{ SD } & \multirow{2}{*}{$\begin{array}{c}\text { Mean } \\
\text { Difference }\end{array}$} & \multicolumn{2}{|c|}{$\begin{array}{c}95 \% \text { Confidence Interval } \\
\text { of the Difference }\end{array}$} & \multirow[t]{2}{*}{$\mathbf{t}$} & \multirow[t]{2}{*}{ df } & \multirow{2}{*}{$\begin{array}{c}\text { p- } \\
\text { value }\end{array}$} \\
\hline & & & & & & \begin{tabular}{|l|} 
Lower \\
\end{tabular} & Upper & & & \\
\hline \multirow{2}{*}{$\begin{array}{c}\text { Harris Hip Score At } 1 \text { Year } \\
\text { Follow Up }\end{array}$} & Short & 20 & 85.75 & 7.38 & \multirow{2}{*}{-4.00} & \multirow{2}{*}{-7.66} & \multirow{2}{*}{-0.34} & \multirow{2}{*}{-2.22} & \multirow{2}{*}{38} & \multirow{2}{*}{$0.03^{*}$} \\
\hline & Long & 20 & 90.75 & 5.92 & & & & & & \\
\hline
\end{tabular}

Independent sample t test $* p<0.05$ statistically Significant, $p>0.05$ Non Significant, NS

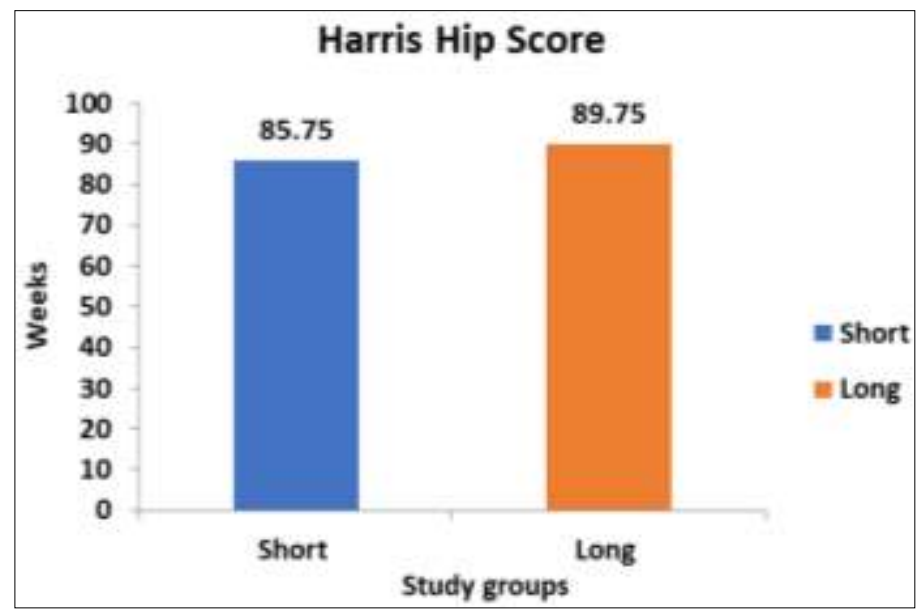

Chart 3: graph showing modified harris hip score at 1 yr follow up 
Results according to modified Harris hip score in short PFN group

Table 5: Results according to modified harris hip score in short pfn group

\begin{tabular}{|c|c|c|}
\hline Variable & Frequency & Percentage \\
\hline Excellent & 9 & 45 \\
\hline Good & 8 & 40 \\
\hline Fair & 2 & 10 \\
\hline Poor & 1 & 5 \\
\hline
\end{tabular}

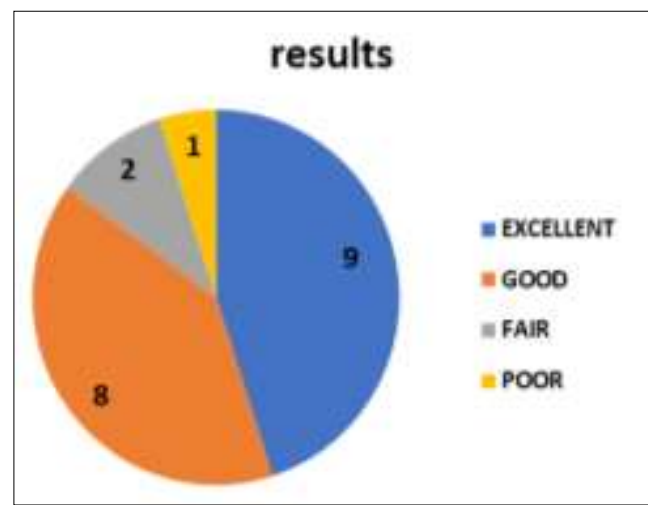

Chart 4: graph showing results according to modified hhs in short pfn group
Results according to modified Harris hip score in long pfn group

Table 6: results according to modified harris hip score in long pfn group

\begin{tabular}{|c|c|c|}
\hline Variable & Frequency & Percentage \\
\hline Excellent & 8 & 40 \\
\hline Good & 9 & 45 \\
\hline Fair & 3 & 15 \\
\hline Poor & 0 & 0 \\
\hline
\end{tabular}

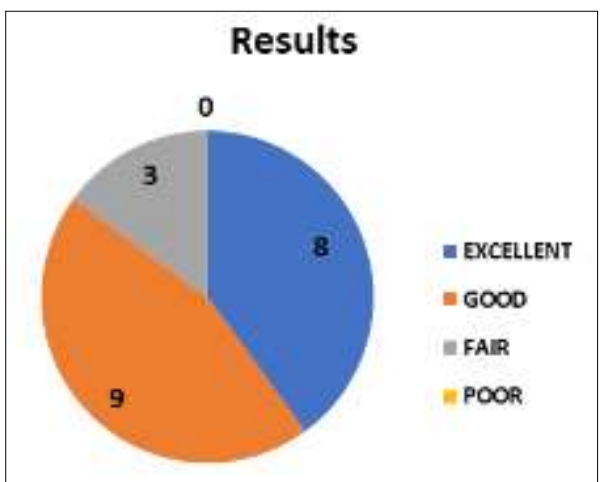

Chart 5: graph showing results according to modified hhs in long pfn group

\section{Complications}

Table 7: table showing complications

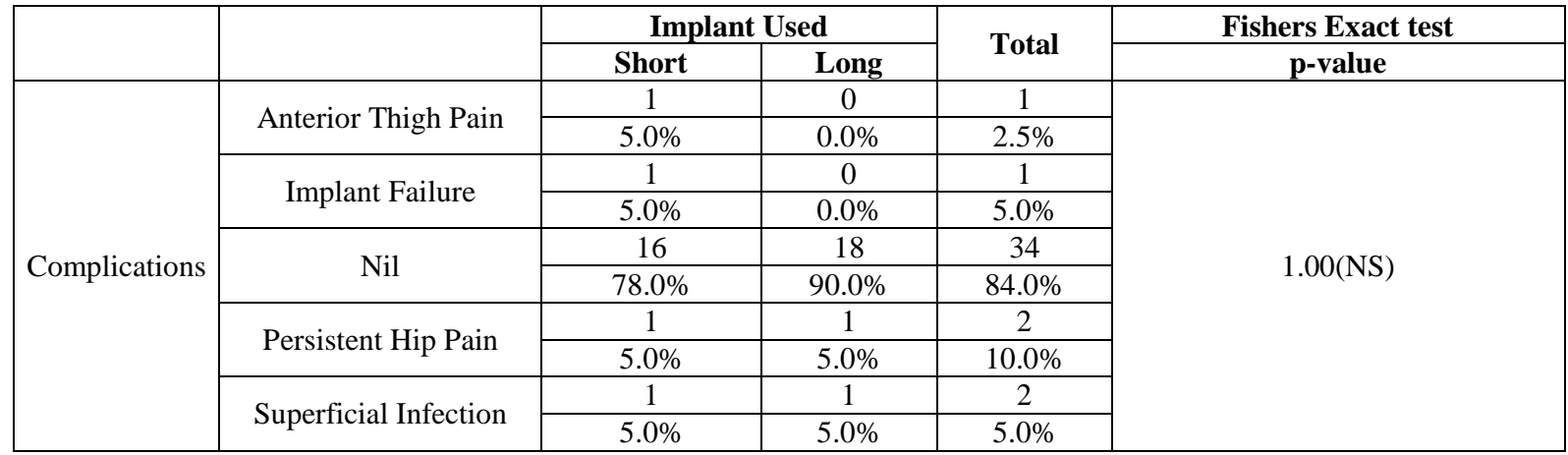

$* p<0.05$ statistically Significant, $p>0.05$ Non Significant, NS

Case 1: 47 year old female with history of slip and fall at home sustained right type 2 intertrochanteric fracture

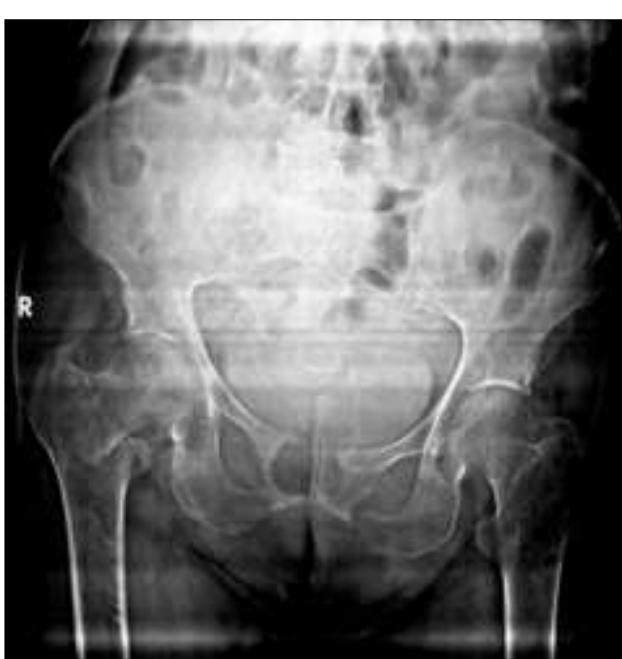

Pre Operative Radiograph

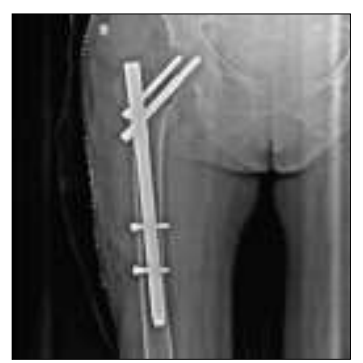

Post Operative Radiograph

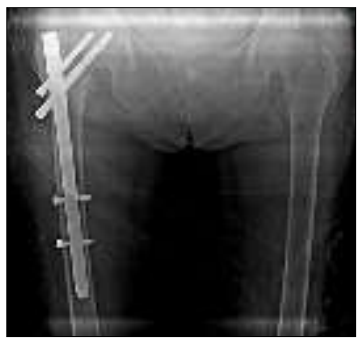

3 Months Follow Up 


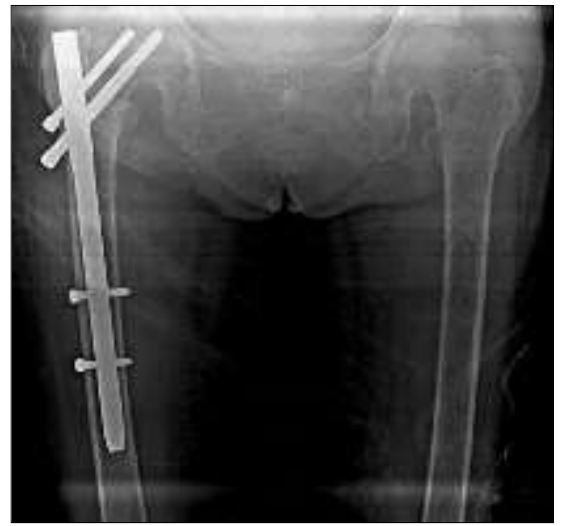

6 Months Follow Up

\section{Clinical Images}
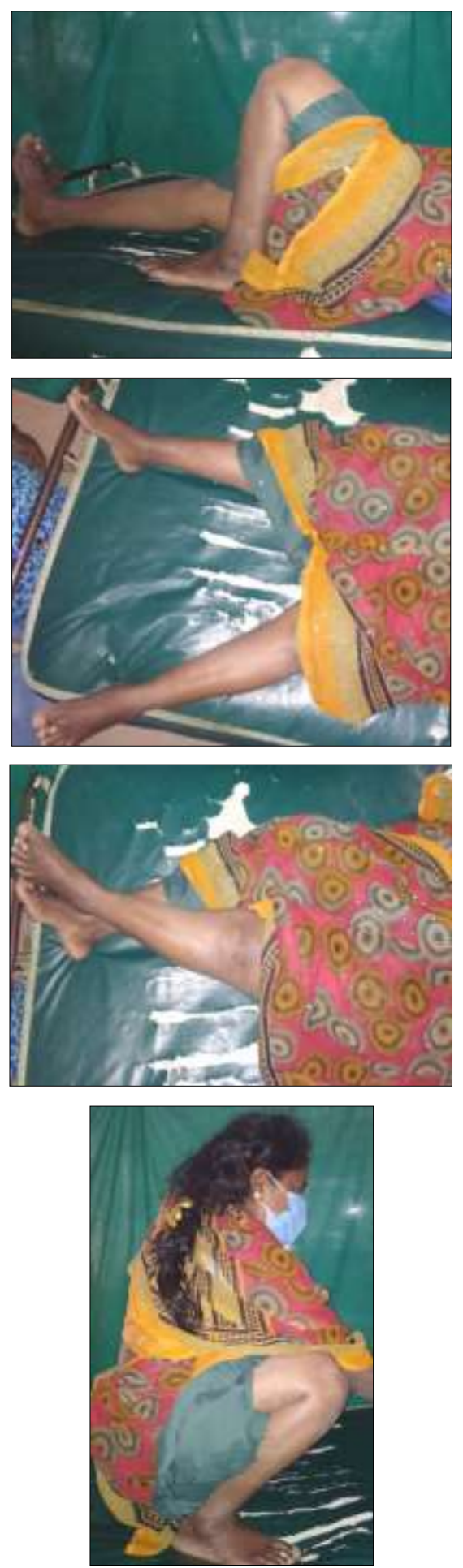

Case 2: 40 year old male patient with h/o slip and fall, treated with long pfn

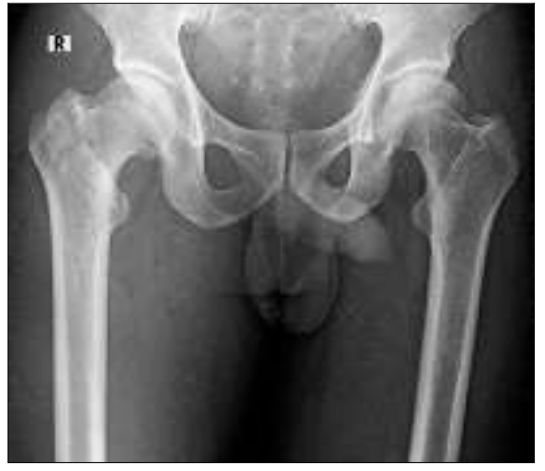

Pre Operative Radiograph

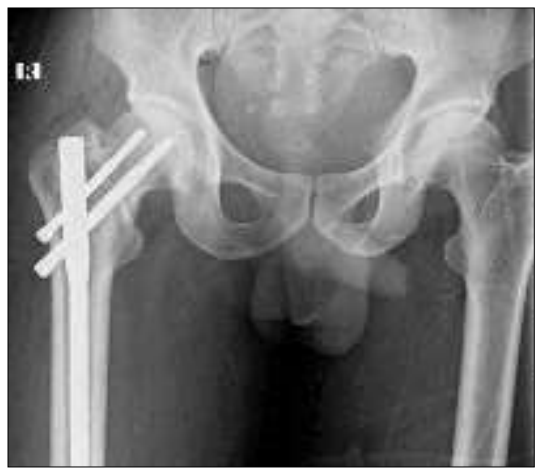

Post Operative Radiograph
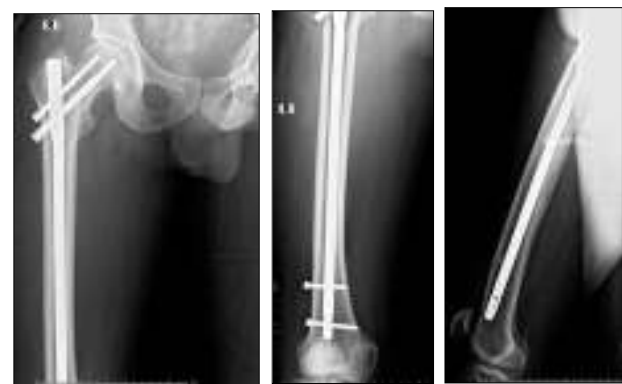

Post Operative Radiographs
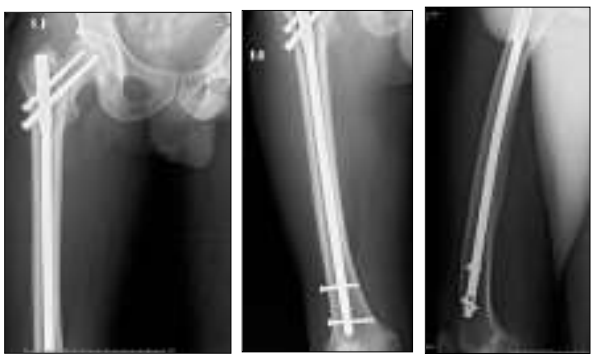

3 Months Follow Up Radiograph
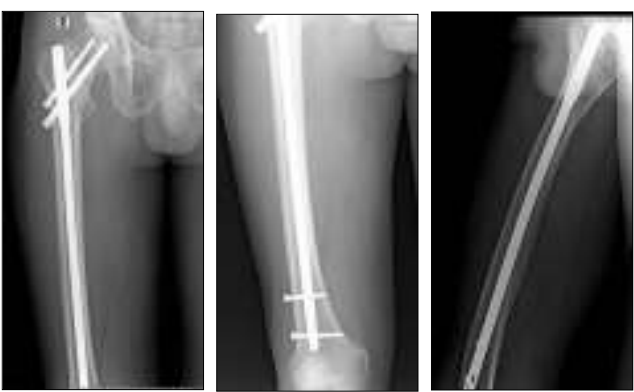

6 Months Follow Up Radiograph 


\section{Functional Outcome At 1 Year Follow Up}
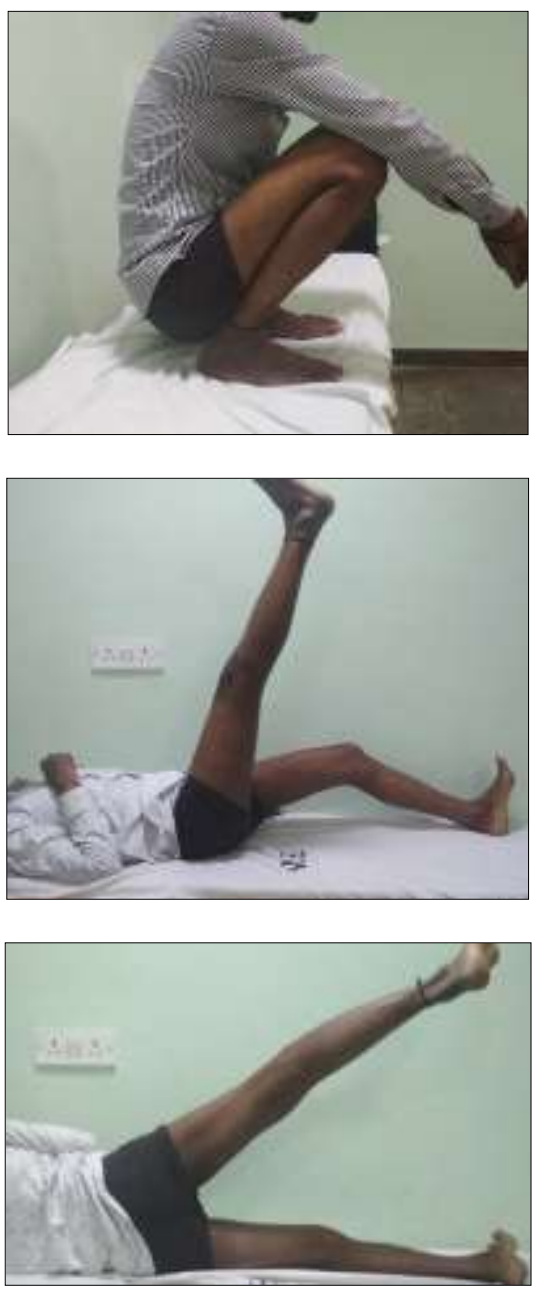

\section{Discussion}

The successful treatment of proximal femur fractures depends on many factors like: Age of the patient, general health, time from fracture to treatment, the adequacy of treatment, concurrent medical illness, stability of fixation.

Our study was conducted on 40 patients with stable intertrochanteric femur fracture, out of which 20 were treated with short PFN and 20 were treated with long PFN.

In our study, patients age group ranged from $18-80$ years. In short PFN group, $2(10 \%)$ were between $31-40$ years, $3(15 \%)$ were between $41-50$ years, $8(40 \%)$ were between 51-60 years, $4(20 \%)$ were between 61-70 years, $2(10 \%)$ were between 71-80 years. In long PFN group, 3 (15\%) were between 31-40 years, $3(15 \%)$ were between 41-50 years, 6 $(30 \%)$ were between $51-60$ years, $4(20 \%)$ were between 61 70 years, 4 (20\%) were between 71-80 years.

In short PFN group $15(75 \%)$ were males and $5(25 \%)$ were females. In long PFN group $10(50 \%)$ were males and $10(50 \%)$ were females.

$10(50 \%)$ patients were operated on right side and $10(50 \%)$ patients were operated on left side in short PFN group. 14 (70\%) patients were operated on right side and $6(30 \%)$ patients were operated on left side in long PFN group.

16 cases $(80 \%)$ affected were due to direct impact following trivial fall, 4 cases (20\%) due to road traffic accident in short PFN group, 18 cases (90\%) affected were due to direct impact following trivial fall, 2 cases $(10 \%)$ due to road traffic accident in long PFN group.

According to the Boyd and Griffin classification, out of 20 cases in short PFN group 7 (35\%) cases were of type 1, 13
(65\%) were of type 2 and out of 20 cases in long PFN group $11(31 \%)$ were of type 1 and $9(45 \%)$ were of type 2 .

All the patients were evaluated for associated medical problems and were referred to respective department and treated accordingly. Associated injuries were evaluated and treated simultaneously. The patients were operated on elective basis after overcoming the avoidable anesthetic risks.

Short PFN took a mean operative time of 50.50 minutes while in long PFN group mean operative time was 55.15, which was longer compared to short PFN group. There was no statistical significance between mean intra operative blood loss of long and short PFN group. Mean blood in loss short PFN group was $94 \mathrm{ml}$ and in long PFN group was $98 \mathrm{ml}$. Mean duration of hospital stay was 9.05 days for short PFN group and 8.75 days for long PFN group.

Patients of both groups were followed up regularly 6 weeks, 12 weeks, 6 months, 12 months.

In our study the mean time for union was 14.9 weeks in short PFN group and 16.05 weeks in long PFN group. Mean Harris hip score in short PFN group at 1 year follow up was 85.75 and score for long PFN group at 1 year follow up was 90.75 which had a statistically significant difference.

In short PFN group 9 (45\%) patients had excellent outcome, 8 $(40 \%)$ patients had good outcome, $2(10 \%)$ patients had fair outcome, $1(5 \%)$ patient had poor outcome. In long PFN group 8 (40\%) patients had excellent outcome, 9 (45\%) patients had good outcome, $3(15 \%)$ patients had fair outcome.

In our study among short PFN group, $1(5 \%)$ patient had anterior thigh pain, $1(5 \%)$ patients had implant failure i.e, peri implant fracture, which required implant removal and exchange nailing with PFN-A, 1(5\%) patient had persistent hip pain, $1(5 \%)$ patient had superficial wound infection which was treated with culture specific antibiotic.

Among long PFN group, 1(5\%) patient had persistent anterior knee pain, 1(5\%) patient had persistent hip pain, $1(5 \%)$ patient had superficial wound infection which was treated with culture specific antibiotic.

Our results are consistent with a study by Hou $\mathrm{Z}$ et al. who concluded that there were no significant difference between the two treatment modalities, complication and reoperation rates for the 2 groups. Treatment with a long nail showed increase in procedure time and blood loss ${ }^{[21]}$.

A retrospective study by Boone et al. conducted in 2014 concluded that, statistically significant lower operative time, Blood loss, and transfusion rate were found in this study for short intramedullary nails. There were no differences seen in length of stay or peri implant fracture. The incidence of peri implant fracture and implant failures were very low in both cohorts which is similar to our results ${ }^{[22]}$.

However, a retrospective study conducted by Zhi Li et al. concluded that the long nail group had significantly lesser failure rate and hip pain rate than those with short nail. But the operative time was significantly longer in the former than the latter intra-medullary device. This was comparable to our study where mean operative time for long PFN group was longer than that of short ${ }^{[23]}$.

A study conducted by Nicholas B Frisch $e t$ al. came up with the result that short nails had the advantage of a faster surgery and lesser blood loss but had a higher rate of peri-implant fractures as compared to longer intramedullary nails. We had one patient in short PFN group with peri implant fracture ${ }^{[24]}$. A study conducted by Xue-Feng Guo et al. concluded that both the intramedullary long and short nail fixation has a good clinical effect in treating intertrochanteric femur fractures in 
the elderly. They showed no significant difference in terms of therapeutic effect, hospital stay and postoperative complications. The incidence of peri implant fractures treated by either length of nails was low. The same results were found in our study ${ }^{[25]}$.

AO foundation recommends that a multi fragmentary intertrochanteric fracture without distal extension or without another fracture distally can be treated with a short intramedullary nail. Preoperatively the anterior bow of the femur of the uninjured extremity needs to be checked. If the tip of the nail comes to lie at the apex of the anterior bow, a long nail or a plate should be used instead.

\section{Conclusion}

This Prospective comparative study between Long and short PFN was performed to assess the functional and radiological outcome in the treatment of stable intertrochanteric fractures.

The following results were drawn from of our study:

- Short PFN took a lower operative time and less intra operative blood loss as compared to a long PFN

- Post operatively incidence of anterior thigh pain was found in short PFN group.

- In terms of final Harris hip score at 1 year of follow up there was statistically significant difference in outcome between short and long PFN groups. Long PFN group had a better outcome compared to short PFN group.

- There was an incidence of peri implant fracture in short PFN group which required revision surgery. A longer Follow-up would be necessary to draw conclusions with respect to the peri implant fracture rate in the two groups.

- The outcomes of Short and Long PFNs are comparable in terms of function and complication rate with long PFN having statistically significant better functional outcome compared to short group.

Operating with PFN has distinct advantages. Early mobilization and weight bearing is allowed in patients treated with both short and long PFN thereby decreasing the incidence of bedsores, lung infections, deep vein thrombosis. Thorough preoperative planning and correct surgical technique, adequate reaming of the femoral canal, insertion of implant and meticulous placement of distal locking screws and early post-operative rehabilitation is essential for successful outcome.

Hence we conclude, long PFN is effective treatment modality for stable intertrochanteric fractures, providing excellent functional outcome and regaining the pre-fall ambulatory status and avoids complications like periprosthetic fracture and anterior thigh pain which is found in short PFN group. However, proper operative technique is important for achieving fracture stability and to avoid major complications. The limitation of our study is the lack of a long term followup preferably 5 years or more and the heterogeneity of the cases in terms of age and associated co-morbidities. Also it is a single centre study.

\section{References}

1. A treatise on dislocations, and on fractures of the joints [Internet]. Welcome Collection. [Cited 2020 Sep 17]. Available from: https://wellcomecollection.org/works/vhg2uuau

2. Asif N, Ahmad S, Qureshi OA, Jilani LZ, Hamesh T, Jameel T. Unstable Intertrochanteric Fracture Fixation-Is Proximal Femoral Locked Compression Plate Better Than Dynamic Hip Screw. J Clin Diagn Res
2016;10(1):RC09-RC13.

3. Kumar N, Kataria H, Yadav CS, Gadagoli BS, Raj R. Evaluation of proximal femoral locking plate in unstable extracapsular proximal femoral fractures: Surgical technique and mid term follow up results. Journal of clinical orthopaedics and trauma, 2014, 137-45. [PMC free article] [PubMed - Google Search [Internet]. [Cited 2020 Sep 17].

4. Halder SC. The Gamma nail for peritrochanteric fractures. J Bone Joint Surg Br 1992;74(3):340-4.

5. Leung KS, So WS, Shen WY, Hui PW. Gamma nails and dynamic hip screws for peritrochanteric fractures. A randomised prospective study in elderly patients. J Bone Joint Surg Br 1992;74(3):345-51.

6. Weil YA, Gardner MJ, Mikhail G, Pierson G, Helfet DL, Lorich DG. Medial migration of intramedullary hip fixation devices: a biomechanical analysis. Arch Orthop Trauma Surg 2008;128(2):227-34.

7. Pajarinen J, Lindahl J, Michelsson O, Savolainen V, Hirvensalo E. Pertrochanteric femoral fractures treated with a dynamic hip screw or a proximal femoral nail: A Randomised Study Comparing Post-Operative Rehabilitation. The Journal of Bone and Joint Surgery British volume 2005;87-B(1):76-81.

8. Boriani S, Bettelli G, Zmerly H, Specchia L, Bungaro P, Montanari $\mathrm{G}$ et al. Results of the multicentric Italian experience on the Gamma(TM) nail: A report on 648 cases. Orthopedics 1991;14(12):1307-14.

9. Germonville TH, Lecestre P, Laimouche A. The SOR Peritrochanteric fractures treated by gamma nailing: study of 300 cases. Eur J Orthop Surg Traumatol 1997;7:255-261. Google Search [Internet]. [Cited 2020 Sep 17].

10. Domingo L, Cecilia D, Herrera A, Resines C. Trochanteric fractures treated with a proximal femoral nail. International Orthopaedics (SICOT) 2001;25(5):298-301.

11. Boldin C, Seibert FJ, Fankhauser F, Peicha G, Grechenig W, Szyszkowitz R. The proximal femoral nail (PFN)-a minimal invasive treatment of unstable proximal femoral fractures. A prospective study of 55 patients with a follow-up of 15 months. Acta Orthop Scand 2003;74(1):53-58. Google Search [Internet]. [cited 2020 Sep 17].

12. Morihara T, Arai Y, Tokugawa S, Fujita S, Chatani K, Kubo T. Proximal femoral nail for treatment of trochanteric femoral fractures. J Orthop Surg (Hong Kong) 2007;15(3):273-7.

13. Schipper IB, Steyerberg EW, Castelein RM, Van der Heijden FHWM, Den Hoed PT, Kerver AJH et al. Treatment of unstable trochanteric fractures. Randomised comparison of the gamma nail and the proximal femoral nail. J Bone Joint Surg Br 2004;86(1):86-94.

14. Min WK, Kim SY, Kim TK, Lee KB, Cho MR, Ha YC et al. Proximal femoral nail for the treatment of reverse obliquity intertrochanteric fractures compared with gamma nail. J Trauma 2007;63(5):1054-60.

15. Adams CI, Robinson CM, Court-Brown CM, McQueen MM. Prospective randomized controlled trial of an intramedullary nail versus dynamic screw and plate for intertrochanteric fractures of the femur. J Orthop Trauma 2001;15(6):394-400.

16. Chen T, Li K, Wang X, Lan H, Zhang J. [Revision cause and effect of gamma nail fixation]. Zhongguo Xiu Fu Chong Jian Wai Ke Za Zhi 2010;24(1):78-81. 
17. Osnes EK, Lofthus CM, Falch JA, Meyer HE, Stensvold I, Kristiansen IS et al. More postoperative femoral fractures with the Gamma nail than the sliding screw plate in the treatment of trochanteric fractures. Acta Orthop Scand 2001;72(3):252-6.

18. Hesse B, Gächter A. Complications following the treatment of trochanteric fractures with the gamma nail. Arch Orthop Trauma Surg 2004;124(10):692-8.

19. Tyllianakis M, Panagopoulos A, Papadopoulos A, Papasimos S, Mousafiris K. Treatment of extracapsular hip fractures with the proximal femoral nail (PFN): long term results in 45 patients. Acta Orthop Belg 2004;70(5):444-54.

20. Parmar DS, Porecha MM, Chudasama SL. Long proximal femoral nails versus short proximal femoral nails for the management of proximal femoral fractures: a retrospective study of 124 patients. Eur J Orthop Surg Traumatol 2011;21(3):159-64.

21. Hou Z, Shi J, Ye H, Pan Z. Treatment of unstable intertrochanteric fractures with percutaneous non-contact bridging plates. Int J Surg 2014;12(5):538-43.

22. Boone C, Carlberg KN, Koueiter DM, Baker KC, Sadowski J, Wiater PJ, et al. Short versus long intramedullary nails for treatment of intertrochanteric femur fractures (OTA 31-A1 and A2). J Orthop Trauma. 2014;28(5):e96-100.

23. Li Z, Liu Y, Liang Y, Zhao C, Zhang Y. Short versus long intramedullary nails for the treatment of intertrochanteric hip fractures in patients older than 65 years. Int J Clin Exp Med. 2015;8(4):6299-302.

24. Frisch NB, Nahm NJ, Khalil JG, Les CM, Guthrie ST, Charters MA. Short Versus Long Cephalomedullary Nails for Pertrochanteric Hip Fracture. Orthopedics. 2017;40(2):83-8.

25. Guo XF, Zhang KM, Fu HB, Cao W, Dong Q. A comparative study of the therapeutic effect between long and short intramedullary nails in the treatment of intertrochanteric femur fractures in the elderly. Chin $\mathbf{J}$ Traumatol 2015;18(6):332-5. 